Alexander Bogner, Karen Kastenhofer, Helge Torgersen

\title{
Antizipierte Technikkontroversen als Governance-Problem
}

Zusammenfassung: Risiko und Ethik haben sich als dominante Problematisierungsweisen in Technikkontroversen etabliert. Diese Rahmungen sind mit bestimmten Governance-Regimes assoziiert. Im Fall der erst im Entstehen begriffenen, sogenannten emerging technologies wie Nanotechnologie oder synthetische Biologie wird Kontroversialität vielfach antizipiert, ohne dass konkrete Anwendungen, Folgen oder Problematisierungen existieren. Hinter solchen Reaktionen auf antizipierte Zukünfte verbirgt sich die Annahme, dass zukünftige Kontroversen nach dem Muster vergangener gestrickt sein werden. Auf Basis dieser Unterstellungen werden Strategien einer antizipierenden Governance entwickelt. In diesem Artikel wird argumentiert, dass technology governance auf Basis einfacher Analogien zu kurz greift. Die Antizipation von Technikentwicklungen und -kontroversen wird selbst zu einem bedeutenden Einflussfaktor in der Produktion gesellschaftlich-technologischer Zukünfte. Für die beteiligte sozialwissenschaftliche Expertise resultiert daraus ein Funktionswandel: Sie wird vom Schiedsrichter zum Mitspieler.

Schlagworte: Technikkontroversen · Anticipatory Governance · Rahmen · Risiko · Ethik

\section{Governing Anticipated Technology Controversies}

Abstract: In technology controversies, risk and ethics are established as dominant frames to expound the problems of the technology at hand. These frames are associated with particular governance regimes. Regarding so called emerging technologies such as nanotechnology or synthetic biology, a propensity for controversies is often anticipated even before concrete applications, consequences or problem frames exist. It is implicitly assumed that future controversies will follow the pattern of past ones. On this basis, strategies of anticipatory governance are developed. In this article we argue that technology governance based on simple analogies poses an inadequate simplification. Rather, the anticipation of technological developments and controversies becomes an important factor for the production of socio-technological futures. This results in a shift in function of the social scientific expertise involved: from a referee to a player.

Keywords: Technology Controversies · Anticipatory Governance · Frame · Risk · Ethics 
Die gesellschaftliche Kontroversialität von Wissenschaft und Technik scheint aus soziologischer Perspektive zunächst einmal keine neue oder innovative Themenstellung zu sein. Spätestens seit den 1950er Jahren ist mit den Konflikten um Kernenergie und Großchemie die Krisenanfälligkeit eines Modernisierungsprojekts ins öffentliche Bewusstsein getreten, das Aufklärung, technischen und sozialen Fortschritt in Eins setzt und daher auf die Institutionalisierung von Wissenschaft und Technik angewiesen ist (Gehlen 2007). Technikkontroversen sind daher schon bald zu einem dezidierten Gegenstand empirischer Studien und - seltener - der sozialwissenschaftlichen Theoriebildung geworden (vgl. Nelkin 1984, Lau 1991). Auch wenn sich die Mobilisierungs- und Aushandlungsformen geändert haben mögen, geraten doch auch heute bestimmte Forschungs- und Technologieprojekte unter Druck. Es genügt, an den Streit um die Stammzellforschung zu erinnern oder an die andauernden Auseinandersetzungen um die Grüne Gentechnik. Obwohl also einerseits der Glaube recht stabil zu sein scheint, dass Wissenschaft und Technik zur Verbesserung des Lebens, zu Wohlstand, Sicherheit und neuen Wahlfreiheiten beigetragen haben (Gaskell et al. 2006), ist doch andererseits evident, dass konkrete wissenschaftliche Forschungen und Anwendungsoptionen immer wieder Gegenstand öffentlicher Problematisierung und Kritik waren - und werden.

Eine gänzlich neue Perspektive entsteht nun allerdings für die soziologische Forschung, wenn politisches Regieren und Regulieren sich nicht auf real existierende Technikkontroversen um bereits etablierte oder entwickelte Technologien beziehen, sondern antizipierte Kontroversen zum Gegenstand haben. Beobachtbar ist dies derzeit im Bereich der sog. emerging technologies, also in Technologiebereichen, die erst im Entstehen sind bzw. im Hinblick auf ganz disparate Technisierungsprojekte, denen dieser Begriff gewissermaßen als gemeinsame Klammer dient (unter ökonomischer Perspektive Giersch 1982). Angesichts der potenziellen Kontroversialität solcher emergierenden Technologien wie der Nanotechnologie oder der synthetischen Biologie liegt die Überlegung nahe, Lehren aus vergangenen Technikkontroversen für den Umgang mit zukünftigen Kontroversen zu entwickeln. In diesem Sinn fordert Rita Colwell, Direktorin der US-amerikanischen National Science Foundation und somit des größten Fördergebers der USA für Nanotechnologie: "We can't risk making the same mistakes that were made with the introduction of biotechnology", wobei Colwell sich dabei vor allem auf die Kommunikation zwischen Forschung, Technologiepolitik und Öffentlichkeit bezieht. ${ }^{1}$ In ähnlicher Weise äußert sich der britische Wissenschaftsminister David Sainsbury: Die Regierung sei aufgerufen, der Nanotechnologie dasselbe Schicksal wie der Grünen Gentechnik zu ersparen. ${ }^{2}$ 
Hinter solchen Aussagen verbirgt sich offensichtlich die Erwartung, dass antizipierte Technikkontroversen bezüglich Akteurskonstellationen, Mobilisierungs- und Austragungsformen nach dem Muster vergangener Kontroversen gestrickt sein werden. Derartige Analogiebildungen sind freilich problematisch, wie wir im Folgenden zeigen werden, weil sie politisches Handeln auf Basis ungeeigneter Prämissen programmieren. Um dies zu erkennen, bedarf es allerdings eines analytischen Instrumentariums, das systematische Vergleiche zwischen Technikkontroversen erlaubt. Zu diesem Zweck wird im ersten Abschnitt auf den Begriff des Rahmens rekurriert; dies macht deutlich, dass Technologien auf der Ebene ihrer öffentlichen Diskursivierung ganz unterschiedlichen Thematisierungsweisen unterworfen sind (1.). Daran anschließend wird die Frage aufgenommen, welche Konsequenzen sich aus der spezifischen Rahmung von Technikkontroversen für technology governance ergeben (2.). Mit dem Schwenk auf gegenwärtige Debatten um emerging technologies geraten Technologiebereiche in den Blick, die aufgrund der ihnen zugeschriebenen potenziellen Kontroversialität zum Gegenstand vielfältiger politischer Steuerungsbemühungen werden; diese durch ihre spezifische Zeitperspektive geprägten Aktivitäten werden unter dem Begriff anticipatory governance gefasst (3.). Der damit verbundene Governance-Wandel wird im letzten Schritt im Hinblick auf die Rolle einer beratungsorientierten sozialwissenschaftlichen Expertise genauer beleuchtet. Dabei wird deutlich, dass es im Zuge von anticipatory governance zu einem Funktionswandel dieser Expertise kommt, der sich in Form neuartiger Mitgestaltungs- und Verantwortlichkeitsimperative dokumentiert (4.). Am Ende steht ein kurzes Resümee (5.).

In diesem Artikel wird also die These entwickelt, dass Technikkontroversen jeweils spezifische Herausforderungen für technology governance darstellen und daher politische Strategien auf Basis einfacher Analogien zu kurz greifen müssen. Mit Blick auf den Bereich der emerging technologies wird ganz konkret gezeigt, dass sich im Fall antizipierter Technikkontroversen ein Funktionswandel von Expertise vollzieht: Es kommt zu einer strategischen Einbeziehung von ExpertInnen und ForscherInnen in den Prozess der Technikgenese; sie werden Teil eines vorsorglichen politischen Umgangs mit antizipierten Kontroversen, und das heißt gleichzeitig, dass sie zu AdressatInnen veränderter Erwartungen, Aufgaben und Verantwortlichkeiten werden. 


\section{Technikkontroversen und ihre Rahmungen}

Der folgende Abschnitt hat zum Ziel, die Bedeutung von Rahmen (oder Frames) für die analytische Differenzierung von Technikkontroversen zu unterstreichen. Es wird gezeigt, dass Technikkontroversen in ihrer Struktur und Verlaufsform ganz wesentlich durch Rahmen geprägt werden. Diese Einsicht ist für die Diskussion, inwiefern man aus vergangenen für zukünftige (oder antizipierte) Technikkontroversen lernen könne, einigermaßen folgenreich. Denn schon die noch recht schematische Differenzierung von Kontroversen anhand ihrer Rahmungen macht deutlich, dass technology governance auf Basis einfacher Analogiebildungen zwischen Technikkonflikten zu kurz greift.

Im Anschluss an Goffman (1993) verstehen wir Frames als machtvolle Organisationsprinzipien individueller Wahrnehmungen und Deutungen. In Konflikten kommt Frames die Aufgabe zu, eine gemeinsame diskursive Basis für deren Austragung herzustellen. In diesem Sinne stellen Frames so etwas wie gemeinsam geteilte Relevanzen dar, die für die grundsätzliche Ordnung des Diskursverlaufs maßgeblich sind. Auf der Ebene konkreter Bewertungen sind Frames ergebnisoffen: Man kann innerhalb desselben Frames zustimmen oder ablehnen, aus welchen Gründen auch immer (divergierende Interessen, Fundamentalkritik am dominanten Framing etc.). Dies muss deshalb betont werden, weil Frames oft mit konkreten normativen Positionen in der politischen Auseinandersetzung assoziiert werden (z. B. Schön/Rein 1994). Technikkonflikte erscheinen auf diese Weise als Kampf konkurrierender Frames. Eine solche Konzeption ist soziologisch unbefriedigend; sie bleibt hinter Georg Simmels Einsicht zurück, dass Konflikte gemeinsam geteilter Relevanzen bedürfen, um überhaupt ausgetragen werden zu können (Simmel 1958). Ohne derartige Gemeinsamkeiten werden Konflikte nicht sichtbar; es herrscht vielmehr wechselseitige Indifferenz. Für die Entwicklung und diskursive Austragung von Kontroversen sind also die Gemeinsamkeiten zumindest ebenso konstitutiv wie die normativen Differenzen. In diesem Sinne sind Rahmen weder identisch mit normativen Positionen oder Vorannahmen, noch bestimmen sie im Einzelnen über die Tragfähigkeit von Argumenten. Sie legen vielmehr fest, was als Argument gelten kann.

In den großen Technikkontroversen, so die hier vertretene These, waren und sind im Wesentlichen zwei Rahmen dominant: Risiko und Ethik (Bogner 2009, Kastenhofer 2009a). Die Auseinandersetzungen um Kernenergie, Grüne Gentechnik, Mobilfunk oder den Klimawandel sind herausragende Beispiele für Risikokontroversen. ${ }^{3}$ Was charakterisiert solche Kontroversen, 
die in den Kategorien des Risikos ausgetragen werden? Greifen wir als Beispiel die Grüne Gentechnik heraus. Im Mittelpunkt der Kontroverse um die Freisetzung gentechnisch veränderter Pflanzen steht die Frage: Wie hoch ist das Risiko eines Natureingriffs? Welche ökologischen und gesundheitlichen Gefahren resultieren aus dem Versuch, Pflanzen mithilfe der Gentechnik resistenter gegen Schädlinge zu machen? Es wird um Sicherheitsbehauptungen und Risikoeinschätzungen gestritten, nicht um den Wert des Lebens. Damit legt man sich innerhalb dieses Rahmens auf den Problemhorizont der Naturwissenschaften fest. Wichtigster Trumpf in diesen Auseinandersetzungen ist das naturwissenschaftliche ExpertInnenwissen. Nur diejenigen, die ihre Position mithilfe wissenschaftlicher Studien zu begründen wissen, können sich in dieser Debatte auf Dauer behaupten.

Natürlich: Hinter der Kritik an der Grünen Gentechnik stehen nicht nur alternative Risikokalkulationen, sondern auch ganz andere Werthaltungen, etwa ein alternatives Naturverständnis (Gill 2003) oder auch Kapitalismuskritik im Gewande der Ökologie. Das heißt, der Risikodiskurs hat selbstverständlich normative Impulse, so wie auch Risikoanalyse und Risikomanagement niemals wertfrei sind. Doch auf diese "tiefer liegende" Ebene greift unser Frame-Ansatz nicht zu. Es geht uns nicht um die Frage: Was ist der eigentliche Konfliktgrund? Sondern: In welcher Form wird der Konflikt diskursiv bearbeitet? Und auf dieser Ebene der Diskursivierung von Technologien ist klar: Der dominante ExpertInnendiskurs wurde und wird weitgehend als Wissenskonflikt gerahmt. Ein gutes Beispiel dafür bietet das WZBVerfahren zur Herbizidresistenz (van den Daele et al. 1996). Dieser auf Rationalisierung abzielende Deliberationsprozess konnte auf die Frage des Risikos transgener Pflanzen festgelegt werden, weil dies als adäquate Behandlung des Konflikts um Gentechnik galt - auch, zumindest anfangs, unter den Beteiligten. In diesem Sinne ist das WZB-Verfahren als Abstraktion des dominanten Diskurses zu verstehen. Gestritten wurde in erster Linie um ökologische Gefährdungen. Den Kern der Auseinandersetzungen von Expertlnnen und GegenexpertInnen, die an diesem Verfahren teilnahmen, bildeten konfligierende Wahrheitsansprüche. In ähnlicher Weise wurde im Fall der Kernenergie, des Klimawandels oder der BSE-Krise vorwiegend um die Durchsetzung naturwissenschaftlicher Wahrheitsansprüche gerungen. All diese Konflikte waren (und sind) durch die Annahme geprägt, dass auf Basis wissenschaftlicher Expertise und Methodenanwendung über die Tragfähigkeit von Kausalitätsvermutungen und Gefahrenprognosen entschieden werden kann (und muss). Normative Fragen, die nicht als Risikoaspekt reformulierbar sind, bleiben notwendigerweise ausgeklammert. Im Kern der Risikokontroversen geht es um die naturwissenschaftliche Evidenz und damit - 
im Fall von Evidenzstreitigkeiten - um die Qualität des Wissens.

Nun werden heute viele Technikkonflikte mit ausdrücklichem Bezug auf Ethik und Moral verhandelt und nicht allein und primär in Risikobegriffen. Das heißt, Wissenschafts- und Technikkontroversen sind in der jüngsten Vergangenheit mehr und mehr "ethisiert" worden (Lindsey et al. 2001, Maasen 2002). Insbesondere im Streit um die Biomedizin wird die Ethik zur maßgeblichen Reflexions-, Begründungs- und Legitimationsinstanz. In diesen ethisierten Technikkonflikten geht es nicht vorrangig um das richtige Wissen. Der Streit dreht sich vielmehr um das moralisch Gebotene. Zwar behält das Expertlnnenwissen in diesem Kontext weiterhin eine wichtige Funktion (",Sachstandswissen"); es dient jedoch nunmehr als Grundlage für Auseinandersetzungen, in denen um den Grenzverlauf zwischen gut und böse gerungen wird. In diesen Kontroversen steht darum nicht die Qualität des verfügbaren (Expertlnnen-)Wissens im Vordergrund, sondern die Plausibilität normativer Grenzziehungen. Maßgebliche Fragen lauten denn auch: Welche Forschungs- bzw. Technisierungsprojekte sind unter ethisch-moralischen Gesichtspunkten akzeptabel? Welches Wissen wollen wir überhaupt? Und wo sind die Grenzen der Forschung?

In Tabelle 1 sind diese grundlegenden Differenzen zwischen Risiko- und Ethik-Rahmen im Vergleich dargestellt.

Tabelle 1: Risiko- und Ethik-Rahmen im Vergleich

\begin{tabular}{|l|l|l|}
\hline & Risiko & Ethik \\
\hline Streitgegenstand & Das richtige Wissen & Das moralische Gebotene \\
\hline Kernfragen & $\begin{array}{l}\text { Wie hoch ist das } \\
\text { Risiko eines Naturein- } \\
\text { griffs? Sind schädliche } \\
\text { Folgen tatsächlich zu } \\
\text { erwarten? }\end{array}$ & $\begin{array}{l}\text { Wo sind die Grenzen der } \\
\text { Forschung/Technisierung? } \\
\text { Welches Wissen wollen } \\
\text { wir? }\end{array}$ \\
\hline $\begin{array}{l}\text { Basisunter- } \\
\text { scheidung }\end{array}$ & Wahr/Unwahr & Gut/Böse \\
\hline Beispiele & $\begin{array}{l}\text { Kernenergie, Klima- } \\
\text { wandel, Mobilfunk, } \\
\text { Grüne Gentechnik, } \\
\text { Nanotechnologie }\end{array}$ & $\begin{array}{l}\text { Klonen, Gendiagnostik, } \\
\text { human enhancement }\end{array}$ \\
\hline
\end{tabular}

Diese idealtypische Darstellung darf nicht zu dem Missverständnis verleiten, dass die einzelnen Rahmen zwingend mit bestimmten Technologien 
verknüpft wären. Die Technologie determiniert nicht die Art und Weise ihrer Problematisierung. Vielmehr sind Rahmungen immer Resultate vermachteter Aushandlungsprozesse, und zwar über die Form des Diskurses. Zweitens: Die obige Darstellung insinuiert keinesfalls, dass Rahmen in der Praxis in "Reinform" auftreten würden. In Technikkontroversen existieren immer mehrere Rahmen parallel. So wird beispielsweise in der Biomedizin auch in Wissenskategorien gestritten, etwa: Sind Heilungserfolge durch die Stammzellforschung überhaupt zu erwarten? Wie hoch ist der Verbrauch an Eizellen, um geklonte embryonale Stammzellen zu kultivieren? Stellt die IVF ein gesundheitliches Risiko dar? Diese Auseinandersetzungen treten aber hinter die ethischen Auseinandersetzungen um den Status des Embryos und das Wesen der menschlichen Natur zurück. Das heißt, ein bestimmter Rahmen ist dominant, andere sind ihm untergeordnet. Ebenso werden auch in den Risikokontroversen nicht nur Konflikte um das "wahre" Wissen deutlich (also um korrekte Risikoberechnungen und Folgenabschätzungen), sondern auch unterschiedliche Wertsetzungen. Etwa: Ist der Erhaltung der Biodiversität mehr Gewicht beizumessen als dem Ziel der Maximierung des landwirtschaftlichen Ertrags? Andere Rahmen, wie etwa der ÖkonomieFrame (z. B. Verteilungsgerechtigkeit), treten in den Hintergrund, obwohl sie von einzelnen Akteurlnnen zumindest ebenso vorgebracht werden.

Frames sind mit erheblichen Machtwirkungen verbunden. Zum einen steuern und strukturieren Frames unsere Seh-, Denk- und Handlungsgewohnheiten; zum anderen determinieren Frames Kontroversen insofern, als die KontrahentInnen sich konstruktiv auf etablierte Frames beziehen müssen. Für die aktuellen Ethikdebatten heißt das beispielsweise: PolitikerInnen und ForscherInnen können ihr Plädoyer für die Stammzellforschung nicht einfach nur ökonomisch begründen („Standortsicherung“); sie müssen es immer auch ethisch reformulieren. Eine schöne Illustration für die Macht der Frames bietet der folgende Ausschnitt aus einem Interview mit dem ehemaligen deutschen Bundeskanzler Gerhard Schröder. Im Kontext der damals auflebenden Debatte um die Chancen und Risiken der Biotechnologie sagt er dem „Tagesspiegel“:

„Und ich möchte nicht, dass man mir, der ich kein prinzipieller Gegner dieser Technologie bin, unterstellt, dass ich nur unethische Motive hätte. Über die Frage nachzudenken, was man mit Gentechnik an Heilungsprozessen bisher unbekannter Art in Gang setzen kann, ist auch ethische Verantwortung. Ich setze noch einen drauf: Es ist nicht unethisch, darüber nachzudenken, ob man einem Volk in einer entwickelten Industriegesellschaft die ökonomische Nutzung dieser Technologie möglich macht oder nicht. Die Biotechnologie eröffnet uns 
Alexander Bogner, Karen Kastenhofer, Helge Torgersen

wirtschaftliche Chancen, und sie wird ähnlich wie die Kommunikations- und Informationstechnologie in Zukunft die Volkswirtschaft beeinflussen." (Tagesspiegel, $\underline{\underline{7.4 .2001}})^{4}$

Es wird anhand dieser Interviewpassage offensichtlich, dass der Kanzler sich gezwungen sieht, sich positiv auf den dominanten Diskurs der Ethik zu beziehen. Gleichzeitig sensibilisiert dies dafür, wie schwierig es ist, etablierte Rahmungen selbst in Frage zu stellen - auch wenn im Kontext manifester Kontroversen selbstverständlich immer Kämpfe um die Rahmung mitlaufen.

\section{Governance-Regimes}

Was bedeutet es nun für technology governance, wenn Technikkontroversen im Rahmen von Risiko oder Ethik ausgetragen werden? Welche Implikationen hat die spezifische Rahmung von Technikkontroversen für politisches Regieren und Regulieren? Hinter diesen Fragen verbirgt sich die Vermutung, dass die jeweilige Rahmung für den politischen Umgang mit Technikkontroversen einen Unterschied macht. Dies soll im Folgenden anhand ausgewählter Beispiele gezeigt werden. Zuvor jedoch erscheint es angezeigt, etwas genauer auf den allgegenwärtigen Begriff der Governance einzugehen.

Im Prinzip kondensieren im Governance-Begriff Beobachtungen über einen Formwandel des Regierens, der nicht-hierarchische, netzwerkförmig organisierte und kooperative Formen schärfer profiliert. Auch wenn der Begriff aufgrund multidisziplinärer Verwendung bis heute keine festen Konturen gewonnen hat, so lässt sich immerhin für die Sozialwissenschaften ein gemeinsamer Bedeutungskern festhalten. Governance bezieht sich hier auf Formen kollektiver Handlungskoordination, die nicht markt- oder hierarchieförmig organisiert und nicht zentralstaatlich gesteuert sind (Zürn 2007, Mayntz 2009). Es handelt sich um Formen des (Selbst-)Regierens, die sich nicht auf die gesetzliche Regulierung einzelner Aspekte (top-down) beschränken, sondern die verschiedenen relevanten Akteurlnnen (Wissenschaft, Privatwirtschaft, Interessenvertretungen, zivilgesellschaftliche Organisationen etc.) in einer umfassenden Weise in Entscheidungen über neue Technologien einzubinden versuchen. Mögliche Maßnahmen sind somit nicht nur Gesetze und Verordnungen, sondern alle Arten von "soft law" wie z. B. Selbstverpflichtungen, professionelle Richtlinien, Vereinbarungen zwischen Akteurlnnen etc. In diesem Sinne halten Karinen und Guston (2009, S. 219) fest: 
"Governance commonly refers to the move away from a strictly governmental approach to one in which a variety of regulatory activity by numerous and differently placed actors becomes possible without detailed and compartmentalised control from the top".

Die Rede von Governance impliziert im Kern also einen Formwandel des Regierens aufgrund komplexer Interdependenzen und verteilter Steuerung. Ein solcher Ansatz wird unterschiedlich argumentiert: Einerseits soll er einem empfundenen demokratischen Defizit entgegenwirken (Europäische Kommission 2001), andererseits wird der Governance insbesondere in Mehrebenensystemen wie der EU gegenüber einer bloßen Regulierung eine höhere Steuerungseffizienz und die Entlastung staatlicher Institutionen zugesprochen (von Blumenthal 2005). Im Weißbuch der Europäischen Kommission, einem hochrangigen Dokument zur Reform des Europäischen Regierens, wird dementsprechend vorgeschlagen:

„.. . die politische Entscheidungsfindung zu öffnen, und mehr Menschen und Organisationen in die Gestaltung und Durchführung der EU-Politik einzubinden. Es plädiert für mehr Offenheit sowie für eine größere Verantwortung und Rechenschaftspflicht aller Beteiligten." (Europäische Kommission 2001, S. 4)

Im Folgenden soll nun für die Differenzen zwischen risk governance und ethics governance sensibilisiert werden. Dahinter steht die Vorstellung, dass unterschiedliche Rahmen ganz unterschiedliche Erwartungen an Konfliktkonstellationen, institutionelle Architekturen, Politik- und Legitimationsmuster zum Ausdruck bringen - und damit auf unterscheidbare Governance-Strukturen verweisen. ${ }^{5}$ Die Bedeutung der einzelnen Rahmen für das Regieren und Regulieren von Technologie(kontroverse)n wird im Einzelnen aufgeschlüsselt anhand der Dimensionen von 1) Politikberatung 2) Öffentlichkeitsbeteiligung und 3) politischer Legitimation.

1) Nimmt man die Unterscheidung zwischen starker und schwacher Interdisziplinarität zum Ausgangspunkt (Kastenhofer 2009b), so lassen sich auf der Ebene der Politikberatung signifikante Unterschiede zwischen risiko- bzw. ethikspezifischer Expertise herausarbeiten. Diese Differenzen betreffen sowohl die Institutionalisierungs- und Produktionsform von Expertise als auch die an sie gerichteten Qualitätskriterien. Schwache Interdisziplinarität bezeichnet die Kooperation innerhalb von Fakultäten oder Fachkulturen (im Sinne von Snow 1963), also z. B. die Zusammenarbeit zweier Fächer innerhalb der Naturwissenschaften; unter starker Interdisziplinarität verstehen wir Kooperationen über die Grenzen der Fachkulturen hinaus, also etwa die Zusammenarbeit zwischen natur- und sozialwissenschaftlichen 
Disziplinen. Die für Risikokontroversen relevante Expertise ist durch schwache, jene in Ethikdebatten tonangebende Expertise durch starke Interdisziplinarität (oder sogar durch Transdisziplinarität im Sinne der Beteiligung von Laien) gekennzeichnet. Für die biologische Sicherheitsforschung spielt sozial- oder geisteswissenschaftliches Spezialwissen gegenwärtig keine besondere Rolle (und wenn doch, dann wird dieses Wissen in getrennten Foren verhandelt) ${ }^{6}$; hingegen versammeln die in den großen bioethischen Regulierungsdebatten maßgeblichen nationalen Ethikräte in der Regel Vertreterlnnen aller Fachkulturen (Geistes-, Sozial- und Naturwissenschaften sowie der Medizin). Während Ethikexpertise im Modus der Deliberation erstellt wird, verläuft Risikoexpertise im Modus der Forschung. Diese Forschung ist in Deutschland im Rahmen spezifischer Forschungsinstitutionen organisiert, wie etwa dem Robert-Koch-Institut, dem Umweltbundesamt oder dem seit 2002 bestehenden Bundesinstitut für Risikobewertung. Die Erwartungen an diese Risikoexpertise sind - entsprechend den wissenschaftlichen Qualitätskriterien - Objektivität und Reliabilität. Demgegenüber wird man Ethikexpertise kaum nach der Messlatte wissenschaftlicher Wahrheit beurteilen, sondern vielmehr hinsichtlich ihrer Ausgewogenheit: Vor dem Hintergrund des gesellschaftlichen Pluralismus sollte Ethikexpertise eine gewisse weltanschauliche Heterogenität zum Ausdruck bringen. Politische Aufgabe der nationalen Ethikräte ist denn auch allenfalls die Koordinierung des Dissenses - nicht dessen Überwindung. Anders ist dies im Fall der Risikoforschung: Die in Risikofragen gegeneinander anforschenden ExpertInnen leben und arbeiten im Prinzip nach dem Ideal akademischer Wahrheitsfindung.

2) Im Kern des Risiko-Rahmens geht es um Statistik und Wahrscheinlichkeiten, rationales Entscheiden beruht auf naturwissenschaftlicher Expertise. Damit einher geht eine Privilegierung wissenschaftlicher Rationalität und wenigstens im Prinzip - eine Abwertung alternativer Wissensformen. Typisch für risk governance sind standardisierte Verfahren (Benchmarking, Monitoring, Audits) und der Rekurs auf sound science. ${ }^{7}$ Im Risikodiskurs dominiert die Sprache der Ingenieure, es geht um Sicherheit, Kontrolle und Objektivität. Natürlich gibt es auch im Kontext des Risikos Bestrebungen, die Öffentlichkeit zu beteiligen und alternative Rationalitäten aufzuwerten (De Marchi 2003). Doch der Status der Öffentlichkeit bleibt im Kontext des Risikos zumindest ambivalent: Es besteht prinzipiell immer die Möglichkeit, Risikofragen als reine Wissenschaftsangelegenheit auszugeben und abweichende Laienpositionen als "irrational“ und damit aufklärungsbedürftig zu brandmarken. Genau dies erklärt auch das Beharrungsvermögen von Interventionsformen, die primär auf ein traditionelles public understanding of 
science abzielen und als Einwegkommunikation zu Aufklärungszwecken praktiziert werden (kritisch dazu Michael 1996, Irwin 2001).

Demgegenüber ist es in Ethikfragen nicht länger legitim, Laien als aufklärungsbedürftige KlientInnen der Wissenschaft zu konzeptualisieren. Die Durchsetzung des Ethik-Frames formuliert vielmehr den Anspruch einer Laienbeteiligung auf Augenhöhe; wenn es um Wertfragen geht, zählt zwangsläufig die Meinung jedes und jeder Einzelnen. Genau daraus entsteht ein subtiler Zwang zur Öffentlichkeitsbeteiligung, wobei der politische Wille gleichwohl in der Praxis häufig auf ein geringes Partizipationsinteresse stößt. Gerade Bürgerkonferenzen - Experimente zur Beteiligung von Laien, die wir verstärkt zu bioethischen Themen finden (Abels/Bora 2004) - sind viel eher Verfahren, um bislang nicht aktive Vertreterlnnen der schweigenden Öffentlichkeit in einen Partizipationsprozess zu involvieren, denn eine Methode zur Kanalisation oder Befriedung politischer Teilhabebestrebungen. BürgerInnenbeteiligung realisiert sich im Kontext der Ethisierung nicht als Protest, sondern als "Laborexperiment", nämlich in Form wissenschaftlicher Dialogprojekte, die von "Partizipationsprofis" initiiert und organisiert werden - ohne reale Teilhabebestrebungen und individuelle Betroffenheiten, ja meist ohne ein vorgängiges Themeninteresse der Beteiligten (Bogner 2010).

3) Auch im Hinblick auf politische Legitimationsstrategien lassen sich charakteristische Unterschiede zwischen Risiko- und Ethikkontroversen ausmachen. Während im Fall des Risikos - wie erwähnt - das ExpertInnenwissen der entscheidende Bezugspunkt politischer Legitimation ist, werden im Fall ethisierter Konflikte politische Entscheidungen mit Rekurs auf das persönliche Gewissen legitimiert. Dazu zwei Beispiele: Als im Frühjahr 2009 die deutsche Landwirtschaftsministerin die Aussaat einer gentechnisch veränderten Maissorte verbot, hielt sie ausdrücklich fest, sie habe "eine fachlich begründete und keine politische Entscheidung getroffen". ${ }^{8}$ Obwohl die Ministerin eingestand, dass die Skepsis der Bevölkerungsmehrheit gegenüber der Grünen Gentechnik sie nicht kalt gelassen habe, verwies sie in ihrer politischen Entscheidung ausschließlich auf neue wissenschaftliche Erkenntnisse. Zwei experimentelle Studien hätten nachgewiesen, dass "Genmais" der Umwelt schaden könne. ${ }^{9}$

Demgegenüber wird in den großen bioethischen Kontroversen politisches Handeln in den Bereich individueller Wertentscheidung verlagert. Gerade in Debatten um die Embryonenforschung wurde und wird immer wieder betont, wie sehr es sich um eine persönliche Bewertung, eine Gewissensentscheidung handle, vor der die ParlamentarierInnen oder die Regierungen stünden: Nicht Expertlnnenenvernunft oder Parteimoral dienen zur 
Begründung des politischen Votums, sondern Subjektivität und Authentizität. Als Sternstunden des Parlaments gelten in Deutschland noch heute jene Debatten zum deutschen Stammzellgesetz Anfang 2002, in der die ParlamentarierInnen im eigenen Namen sprachen und nach individueller Gewissensüberzeugung abstimmten - ohne auf die Parteidisziplin achten zu müssen. Wer jedoch - wie der britische Premier Gordon Brown im Vorlauf zum neuen Embryonenforschungsgesetz - bioethische Fragen nicht als Gewissensentscheidung freigeben möchte, der sieht sich starker Kritik ausgesetzt, auch aus den eigenen Reihen (BBC News, 25.03.2008). ${ }^{10}$

Die oben ausgeführten, Governance-relevanten Differenzen zwischen Risiko- und Ethik-Frame sind in Tabelle 2 dargestellt.

Tabelle 2: Differenzen zwischen Risk und Ethics Governance

\begin{tabular}{|l|l|l|}
\hline \multicolumn{2}{|l|}{ Risk Governance } & Ethics Governance \\
\hline Politikberatung & $\begin{array}{l}\text { Naturwissen- } \\
\text { schaften }\end{array}$ & $\begin{array}{l}\text { Natur-, Sozial- und } \\
\text { Geisteswissenschaf- } \\
\text { ten; Laien }\end{array}$ \\
\hline Relevante Expertise & $\begin{array}{l}\text { Kommissionsethik } \\
\text { (Ethikräte) }\end{array}$ \\
\hline $\begin{array}{l}\text { Institutionalisierungs- } \\
\text { form }\end{array}$ & $\begin{array}{l}\text { Risikoforschungs- } \\
\text { programme }\end{array}$ & Deliberation \\
\hline $\begin{array}{l}\text { Produktionsform von } \\
\text { Expertise }\end{array}$ & Forschung & Ausgewogenheit \\
\hline Erwartung an Expertise & Objektivität & $\begin{array}{l}\text { Laborexperiment } \\
\text { (Mobilisierung durch } \\
\text { "Partizipationsprofis“) }\end{array}$ \\
\hline Öffentlichkeitsbeteiligung & $\begin{array}{l}\text { Protest (Selbstmobilationsform } \\
\text { lisierung) }\end{array}$ & ja \\
\hline Rahmenkompatibilität & nein & Gewissen \\
\hline Politische Legitimation & ExpertInnenwissen &
\end{tabular}

Die Darstellung verdeutlicht, dass die für die Austragung von Technikkontroversen maßgeblichen Semantiken, eben Risiko oder Ethik, mit spezifischen Ansprüchen an deren politisches Management verknüpft sind, oder kurz: Dass Technikkontroversen als jeweils spezifische Governance-Aufgaben zu verstehen sind. Schon dies dürfte klar machen, dass einzelne Technikkontroversen nicht einfach kommensurabel sind. 


\section{Emerging Technologies und antizipatorische Governance}

Eine völlig neue Situation ergibt sich im Hinblick auf solche Technologiebereiche, die sich eben erst entwickeln und ausdifferenzieren, also den emerging technologies. Darunter werden etwa die Nanotechnologie, die synthetische Biologie ${ }^{11}$ oder neue technologische Entwicklungen im informationsund neurowissenschaftlichen Bereich gefasst. Für sie alle gilt, dass es noch keine entwickelten und diskursiv klar strukturierten Technikkontroversen, etwa zu möglichen Risiken oder ethischen Aspekten, gibt. Vor dem Hintergrund der Erfahrungen mit vergangenen Kontroversen werden solche allerdings erwartet, und es wird auf diese antizipierten Kontroversen sogar im Voraus reagiert.

Womit genau hat man es nun bei diesen sogenannten emerging technologies zu tun? Zwar fehlt eine konzise Begriffsdefinition, es lässt sich aber zusammenfassen, dass darunter (1) ganze Technologiebereiche verstanden werden (im Gegensatz zu konkreten technologischen Anwendungen) und somit ein hohes Abstraktionsniveau besteht, dass (2) die hierunter fallenden Technologiebereiche allesamt Grundlagenforschung und Technologieentwicklung untrennbar verknüpfen und damit auch als Technowissenschaften (im Sinne von Nordmann 2004) bezeichnet werden können, und dass (3) für diese Technologiebereiche die Herausbildung von technologischen Anwendungen in absehbarer Zukunft erwartet oder zumindest angekündigt wird. So fasst der Bericht von Roco und Bainbridge (2002) zusammen, dass wichtige wissenschaftliche Durchbrüche im Kontext der Nano-, Bio-, Informations- und Kognitionstechnologien in den nächsten 10 bis 20 Jahren zu erwarten seien und diese etwa dieselbe Zeitspanne benötigen würden, um in maßgebliche technologische Anwendungen übersetzt zu werden (Roco/ Bainbridge 2002, S. ix).

Diese Eigenschaftskonstellation ist für die Technikregulierung und allgemeiner für den Diskurs über diese Technologien von einiger Bedeutung. Die Vision von gegenwärtig im Entstehen begriffenen technologischen Möglichkeiten, in naher Zukunft absehbaren Anwendungen und daraus resultierenden Kontroversen bringt in besonderer Weise den Faktor Zeit ins Spiel. Sie wirft insbesondere die Frage nach dem „richtigen“ Interventionszeitpunkt auf. Auf diese Frage bezieht sich schon das von Collingridge (1981) formulierte Kontroll-Dilemma. Dieses bestehe, so Collingridge, zwischen einer (zu) früh einsetzenden und darum zwar innovationsrelevanten, aber wenig voraussagefähigen Technikregulierung und einer (zu) spät einsetzenden Technikregulierung, die bereits konkrete Anwendungen vor Augen habe, 
aber eben auch schon vor vollendeten Tatsachen stehe. Emerging technologies ziehen nun freilich erneut die vertraute - und auch dem CollingridgeDilemma implizite - Vorstellung in Zweifel, dass Technikentwicklung ein mehrstufiger, linearer Prozess sein könne. Vannevar Bush hatte 1945 in seinem einflussreichen Bericht "Science: The Endless Frontier" noch die Differenz zwischen Grundlagenforschung und Technologieentwicklung hervorgehoben und einen kausalen Zusammenhang zwischen Investition in Forschung, Förderung von technologischer Innovation und ökonomischer Prosperität postuliert. Auf die neu emergierenden Technowissenschaften lässt sich dieses lineare Modell nicht anwenden, weil Grundlagenforschung und Technologieentwicklung hier kaum noch von einander zu trennen sind. Die Konvergenz der beiden Bereiche betrifft auch die ihnen zugesprochenen Eigenschaften, die postulierte Unsteuerbarkeit und Wertneutralität wissenschaftlicher Innovation und die postulierte Gerichtetheit und Bewertbarkeit technologischer Anwendungsgestaltung. Emerging technologies scheinen beide Eigenschaftskombinationen zu vereinen. Sie gelten als emergent (und damit als nicht direkt planbare Produkte eines komplexen Systems) und zugleich regulierbar, als wertneutral und zugleich bewertungspflichtig.

Zu der erneut virulent gewordenen Frage nach dem richtigen Zeitpunkt regulatorischer Interventionen kommt eine stabilisierte zivilgesellschaftliche Disposition, technologische Innovationen und ihre Steuerung zu problematisieren. Diese "Verringerung der Problematisierungsschwelle" wird von Politik- wie auch (Techno-)Wissenschaftsseite als Vertrauensverlust der Öffentlichkeit gedeutet, sei es in die Selbststeuerung industrienaher Technowissenschaft, in wissenschaftliche Expertise oder auch in staatliche Regulierung. So fasst der Parlamentsausschuss für Wissenschaft und Technologie des britischen House of Lords im Jahr 2000 (und damit knapp nach dem Höhepunkt der BSE-Kontroverse) zusammen, dass die Beziehung zwischen gesellschaftlicher Öffentlichkeit und Wissenschaft (inklusive ihrer technologischen Anwendungen in der Bio- und Informationstechnologie) in einer kritischen Phase stecke. Diese bestünde in einem Vertrauensverlust in wissenschaftliche Regierungsberatung ebenso wie in großem Unbehagen über die enormen Möglichkeiten, die die Wissenschaft gegenwärtig realisiere und die sich abseits der öffentlichen Wahrnehmung und Deliberation zu entwickeln schienen (House of Lords 2000, Absatz 1.1).

Diese Situation mündete - in Verknüpfung mit der wahrgenommenen Konvergenz von Grundlagenforschung und Technologieentwicklung - in die zivilgesellschaftliche Forderung und das politische Versprechen eines upstream engagement, d. h. einer Teilhabe der Öffentlichkeit nicht nur an der Regulierung fertiger technologischer Produkte, sondern bereits an den 
Bedingungen, unter denen technologieassoziierte Grundlagenforschung stattfindet. Allerdings bleibt diese Öffentlichkeitsbeteiligung in Wissenschafts- und Technikfragen sowohl hinsichtlich ihrer Realisierungsformen als auch im Hinblick auf ihre normativen Zielsetzungen reichlich divers: Neben Forderungen nach einer Demokratisierung von Technologiepolitik im Allgemeinen (Sclove 1995, Feenberg 1999) ${ }^{12}$ steht das Ansinnen, qua Partizipation unterschiedliche Perspektiven und Wissensformen zu mobilisieren (Stichwort Transdisziplinarität, vgl. Bogner et al. 2009) ebenso wie Bestrebungen, heiße, unauflösbare Konfliktsituationen und generelle Ablehnung im Voraus zu vermeiden. ${ }^{13}$

Die bislang entwickelte Argumentation macht zweierlei deutlich: Emerging technologies stellen vorwiegend diskursive Phänomene dar und werden auf diese Weise zu Objekten antizipatorischer Governance. Zu vorwiegend diskursiven Phänomenen werden sie zum einen, weil sie als solche (nämlich: als ganze Technologiefelder) markiert und zum Gegenstand von Diskursen werden, zum anderen, weil sie sich zu diesem frühen Zeitpunkt und auf dieser Abstraktionsebene kaum als technischer Gegenstandsbereich definieren lassen (vgl. Selin 2007, Fiedeler 2008). Auch von Seiten der Natur- und Technikwissenschaftlerlnnen werden Begriffe wie Nanotechnologie oder synthetische Biologie zwar in Konferenzankündigungen und Projekteinreichungen bemüht, ihre Definition bleibt aber unklar und ist für die wissenschaftliche Forschungspraxis offenbar auch nicht maßgeblich (vgl. etwa die Suche nach einer adäquaten Definition von Nanotechnologie, Schummer 2006).

Zudem orientieren sich Regulierungsansprüche zunehmend stärker an antizipierten Zukünften als an der Gegenwart und inkludieren eine Vielzahl an Akteurlnnen und Mechanismen. Das Interesse an (antizipierten) Diskursen und Kontroversen über zukünftige Technologien scheint der Steuerung gegenwärtiger technologischer Anwendungen zunehmend den Rang abzulaufen. Man denke in diesem Zusammenhang nur an die Forderung nach einem vision assessment (Grin/Grunwald 2000), einem concern assessment (IRGC 2006), einer primär auf Diskurse bezogenen Technikfolgenanschätzung (Saretzki 1999) oder die Herausbildung einer Sociology of Hopes and Expectations. ${ }^{14}$

Vor diesem speziellen Hintergrund wird gegenwärtig denn auch das Modell einer antizipatorischen Governance wirkmächtig, ${ }^{15}$ insbesondere im Kontext des Ringens um eine good governance der Nanotechnologie. Karinen und Guston (2009) definieren anticipatory governance schlicht als "a distributed form of emerging political order with an emphasis on long-term thinking" (ibid., S. 8), wobei sie - nicht frei von Widersprüchlichkeit - die 
Möglichkeit der Vorhersage zwar in Zweifel ziehen, gleichzeitig aber die Option der Vorbereitung stark machen. ${ }^{16}$ Antizipatorische Governance sei nämlich, in anderen Worten,

„the ability of a variety of stakeholders and the lay-public to prepare for the issues that NSE [nano-scale science and engineering] may present before those issues are manifest or reified in particular technologies" (Karinen/Guston 2009, S. 226).

Diese Zielsetzung könne, und hier greifen Karinen und Guston auf Barben et al. (2008, S. 991 f.) zurück, durch ein großskaliges Forschungsensemble, das sich mit Vorausschau, öffentlicher Beteiligung und der Integration von soziologischen Studien mit Naturwissenschaften und Ingenieurspraxis befasse, verfolgt werden, wenn Fähigkeiten wie kollektive Selbstkritik, Imagination und Lernen aus Versuch und Irrtum eingesetzt würden. Freilich muss antizipatorische Governance als Konzept oder Praxismodell zu diesem frühen Zeitpunkt noch relativ vage erscheinen. Der Versuch, die Governance von Technologien bereits vor deren endgültiger Ausdifferenzierung mitzugestalten, ist aber offensichtlich durch die Erwartung mitgeprägt, dass emerging technologies in Zukunft auf merklichen Widerstand treffen könnten. Es wird die Entwicklung darauf aufbauender Kontroversen und letztlich unauflösbarer Lagerspaltungen - hier Industrie und Forschung, dort BürgerInnen und zivilgesellschaftliche NGOs - antizipiert. Arie Rip (2006) spricht in diesem Zusammenhang recht drastisch von einer nanophobia-phobia (also der Antizipation einer Angstreaktion der Öffentlichkeit auf Ankündigungen nanotechnologischer Innovation) sowie von der Antizipation eines Wow-Yuck Pattern (als einer Lagerspaltung in extrem positive und extrem negative Reaktionen). Er postuliert, dass diese Erwartungshaltungen auf folk theories zurückgingen und nicht auf soziologisch nachgewiesene Evidenz, dass sie allerdings das Verhalten der technowissenschaftlichen Akteure selbst beobachtbar prägten.

Blickt man in der Geschichte der Technikkontroversen ein paar Jahrzehnte zurück, so wird schnell ersichtlich, dass die frühzeitige Problematisierung neuartiger Technologien kein prinzipielles Novum ist. So entzündeten sich rund um die potenziellen Risiken der Arbeit mit rekombinanter DNA im Labor bereits zu einem relativ frühen Zeitpunkt Kontroversen um die Biotechnologie - das Stichwort lautet Asilomar-Konferenz. Allerdings, und das ist nun allerdings entscheidend: Diese Kontroversen blieben zu diesem frühen Zeitpunkt noch auf Expertenzirkel beschränkt (Nisbet/Lewenstein 2002). Die sehr konkreten Risikohypothesen und klaren Evidenzregeln innerhalb der wissenschaftlichen Gemeinschaft erlaubten zudem eine (vor- 
erst) relativ rasche Bearbeitung und Schließung der Kontroverse (auch wenn einige warnende Stimmen nie ganz verstummten). In der breiten Öffentlichkeit wurde die Technologie erst relativ spät und unerwartet Thema; der Höhepunkt der Kontroverse setzte zu einem Zeitpunkt ein, an dem Produkte der Agrobiotechnologie bereits auf dem Markt waren. Dieser Konflikt war außerdem im Wesentlichen auf Europa beschränkt und eng an andere mediale Ereignisse gekoppelt, wie z. B. den Bericht über das Klon-Schaf Dolly und die von Großbritannien ausgehende BSE-Krise Ende der 1990er Jahre, (Bauer/Gaskell 2002).

Die neu emergierenden Technologien hingegen werden bereits zu einem sehr frühen Zeitpunkt als Auslöser potenzieller öffentlicher Kontroversen verstanden und somit zu einem legitimen Gegenstand antizipierender Governance-Maßnahmen. Da jedoch aber weder die tatsächliche weitere Entwicklung der Technologie und ihrer konkreten Anwendungen, noch die öffentlichen Akteurskonstellationen und Mobilisierungsformen vorauszusehen sind, wird unter anderem auf Prognosen zurückgegriffen, die sich qua Analogiebildungen auf die vergangenen Entwicklungen stützen (s. Einleitung). Es ist allerdings fraglich, inwieweit Vergleiche mit vergangenen Technologieentwicklungen und Technologiekontroversen tragfähig sind (vgl. das Postulat der folk theories bei Rip 2006).

Im Gegensatz zu etablierten technologischen Anwendungen sind emerging technologies nur in Umrissen erkennbar. Sie "leben" vor allem in und von Darstellungen (und Gegendarstellungen), die sich an konkrete Fördergeber, potentielle AbnehmerInnen oder die breite Öffentlichkeit wenden (wie Forschungsprogramm-Ausschreibungen, Einreichungen zu Innovationswettbewerben oder öffentlichen PR-Maßnahmen). Eine Konferenzankündigung zum Thema Nanotechnologie aus dem Jahr 2007 verspricht etwa "die weltverändernde Macht der Nanotechnologie zu feiern" ${ }^{17}$ Die sogenannten konvergierenden Technologien werden angekündigt als eine Entwicklung, die eine flexible Manipulation von Bits, Atomen, Neuronen und Genen und damit von Information, Materie, Gehirn und dem Code des Lebens erlaubt. ${ }^{18}$ Ein Spiegel-Artikel zur Zukunft der Biowissenschaften titelt: "Synthetische Biologie. Frankensteins Zeit ist gekommen“ ${ }^{19}$ Dem Machbarkeits- und Fortschrittsdiskurs auf der einen Seite stehen Warnungen in Form von Risiko- oder Ethikdiskursen auf der anderen Seite gegenüber, ohne dass zu klären wäre, wie sich der diskutierte Technologiebereich definieren lässt und welche konkreten Artefakte und Anwendungssituationen in absehbarer Zeit zu erwarten sind. Dies erklärt die Orientierung an vergangenen Technikkontroversen. Doch angesichts der starken Dissoziation der emerging technologies auf Anwendungs- oder Produktebene ist eigentlich noch nicht 
einmal klar, inwieweit traditionelle Kontroversen vom Typ des Gentechnikoder Kernenergiestreits überhaupt zu erwarten sind. Noch unklarer ist, welche Rahmungen solche Kontroversen annehmen würden, und welche Governance-Erfordernisse sich aus diesen Rahmungen ergäben.

\section{Zum Funktionswandel von Expertise}

Im Folgenden gehen wir detaillierter auf die Frage ein, welche Folgen anticipatory governance für beratungsorientierte Expertise hat. Damit greifen wir auf die Ausführungen im zweiten Abschnitt zurück, in dem bereits die recht divergierenden Formen und Funktionen von Expertise in unterschiedlichen Governance-Regimes skizziert wurden. Mit Blick auf die in antizipierten Kontroversen um emerging technologies spezifische Rolle von sozialwissenschaftlicher Expertise lässt sich - so die zentrale These - von einem Funktionswandel von Expertise sprechen. Die Beschreibung eines solchen Funktionswandels vermag gleichzeitig zu einer Präzisierung des Begriffs der anticipatory governance beizutragen. In unserer Analyse beziehen wir uns insbesondere auf das Feld sozialwissenschaftlicher Technikforschung, wie es in Form der Technikfolgenabschätzung (TA), der ELSI-Begleitforschung (Ethical, Legal and Social Implications) bzw. - im weiteren Zusammenhang den Science and Technology Studies (STS) repräsentiert wird. Anhand dieser Beispiele lässt sich der enge Zusammenhang zwischen dem Funktionswandel von Expertise und dem Wandel von Rahmungen illustrieren.

Die Institutionalisierung der TA in den 1970er und frühen 1980er Jahren lässt sich als Folge der Interpretation und Verhandlung maßgeblicher PolicyProbleme im Bereich von Wissenschaft und Technik als Risikofragen verstehen. Die TA ist gewissermaßen Zeugnis dafür, dass man zur Konfliktlösung auf besseres oder problemadäquateres Faktenwissen setzte - ein Kennzeichen von Risikokontroversen. Die TA, so die gängige Erwartung, sollte die Politik mit umfassenden objektiven Informationen über die ökonomischen, ökologischen und sozialen Folgen neuer Technologien im Sinne einer Frühwarnfunktion versorgen - und damit gleichzeitig jenes Expertlnnenwissen liefern, das als legitime Grundlage politischer Entscheidungen in Risikofragen gilt. Diese Erwartungen spiegeln sich beispielhaft in der Einrichtung des Office of Technology Assessment (OTA) in den USA im Jahre 1972 wider (Porter 1995). ${ }^{20}$

Das bekannte „Expertendilemma“ (Nennen/Garbe 1996), d. h. die Selbstrelativierung der ExpertInnen infolge widersprüchlicher Gutachten, indi- 
zierte allerdings bald die brüchige Autorität einer sich als objektiv verstehenden Expertlnnenrationalität. Auch hinsichtlich einer interdisziplinären Abschätzung von Technikfolgen ließ sich der Glaube an eine objektive Wahrheit nicht aufrecht erhalten. Die damit einher gehende Unsicherheit ließ die Bedeutung normativ geprägter Aushandlungsprozesse wieder stärker hervor treten. So wurde von TA die Berücksichtigung von Argumenten (etwa Sozialverträglichkeit) eingefordert, die gesellschaftliche Wertentscheidungen betrafen, wissenschaftlich aber als nicht handhabbar galten (van den Daele 1993). In der Folge versuchte man, weitere Akteurlnnen und Interessen - etwa Stakeholder, aber auch Laien - in den Abschätzungsprozess einzubinden und Ausgewogenheit durch externe Ergebniskontrolle zu gewährleisten (van Eijndhoven 1997).

Diese Krise des wissenschaftlichen Wahrheitsmonopols manifestierte sich u. a. in unterschiedlichen Implementierungsformen von TA im Europa der 1980er Jahre. In Dänemark und den Niederlanden etablierte sich eine „Öffentliche TA“ (van Eijndhoven 1997) mittels Konsensus- oder Bürgerlnnenkonferenzen. Die kognitiven Grundlagen wurden zwar weiterhin von Expertlnnen in Hearings geliefert, die Bewertung aber kam von Laien (Joss 1995). Damit kündigte sich eine Änderung des Diskursrahmens an: Es ging nun nicht mehr vorrangig um die Expertlnnenfrage nach der Evidenz eines Risikos und folglich um die Bestimmung eines richtigen, kognitiv überlegenen Wissens. Vielmehr wurde von den Laien eine Bewertung davon erwartet, was als moralisch inakzeptabel, vertretbar oder geboten zu gelten hätte. Die Plausibilität eines Risikos wurde zu einem Teilaspekt der übergeordneten Frage nach der Akzeptabilität im Lichte von Interessenlage, Nutzenverteilung und Betroffenheit. Mit anderen Worten: Anstelle der ExpertInnendomäne des Wissens trat die den Laien zugesprochene Domäne der Moral in den Vordergrund. Der Fokus der Debatte verschob sich somit von Wahrheit auf Werte. Das ist es, was oben unter dem Begriff der Ethisierung gefasst wurde.

Somit entspricht eine spezifische Form von TA immer auch einer dominanten Rahmung des bearbeiteten Problems. Während eine expertInnenbasierte Folgenabschätzung die kognitive Frage nach der (wissenschaftlichen) Wahrheit bzw. nach der Plausibilität einer Risikobehauptung in den Mittelpunkt stellt, kreist eine partizipative TA unter Laienbeteiligung typischerweise um die Frage nach der moralischen Richtigkeit. Im Rahmen der partizipativen Wende tritt die TA vorrangig als Organisator und zuweilen auch als Beobachter von Deliberationsprozessen in Erscheinung (Abels 2009). $\mathrm{Ob}$ man dies als Funktionswandel der TA beschreiben kann, mag offen bleiben. In jedem Fall handelt es sich um eine Ausdifferenzierung von TA-For- 
men und -Funktionen, die auf die Koexistenz unterschiedlicher ProblemRahmungen verweist und mit diesen interferiert.

Offensichtlich wird ein Funktionswandel jedoch im Hinblick auf die antizipatorische Governance von emerging technologies. Denn im Kontext antizipierter Kontroversen übernimmt die TA eine gestaltende Funktion in der Deutung emergierender Technologien: Wenn die Elemente einer Technologie umstritten und die Anwendungsgebiete unklar sind, wenn der Vergleichsmaßstab umstritten und die Präzedenz interpretationsabhängig ist, sind "Folgen" zwar imaginier-, aber nicht identifizierbar. Wenn also erst festzulegen ist, welche Eigenschaften einer Technologie zuzuordnen und in welchem Rahmen diese sinnvollerweise zu diskutieren sind, dann geht es letztlich um die Definition dessen, was eine bestimmte emerging technology überhaupt ausmacht bzw. um die Festlegung einer Problematisierungsperspektive. In solchen Fällen bedarf es nicht nur einer externen Expertise, sondern einer Reflexionsinstanz über gesellschaftsrelevante Themen in situ, in enger Kooperation mit der Wissenschaft.

Auf diese Weise vollzieht sich im Kontext antizipatorischer Governance ein Funktionswandel sozialwissenschaftlicher Expertise: Sie wird - im Prinzip jedenfalls - zu einem politiknahen Akteur, weil sie mit Gestaltungsfragen konfrontiert wird, die die Kapazität reiner Beobachtung überfordern. TA mutiert auf diese Weise - um eine Fußball-Metapher zu bemühen - während des Spiels vom Linienrichter zum Mitspieler, und zwar für alle ZuschauerInnen sichtbar. Damit gehen notwendigerweise neue Verantwortungszuschreibungen einher: Eine auf Beobachtung und Analyse kaprizierte Technikforschung kann ihre qua Wahl der Beobachtungsperspektive realisierte Deutungsmacht invisibilisieren, die Optionenwahl bleibt auf diese Weise vollständig dem Entscheidungsträger überlassen; eine "eingebettete" Sozialwissenschaft jedoch gestaltet die entstehende Technologie ganz offensichtlich mit, und zwar sowohl über die - nunmehr als Interpretation kenntlich gemachten - Interpretationsangebote als auch qua expliziter Definition derjenigen Fragen, die als gesellschaftlich relevant zu gelten haben.

An dieser Stelle gilt es freilich, einen nahe liegenden Einwand zu entkräften. Macht denn nicht bereits das Programm einer Constructive TA (CTA) Technikgestaltung zu ihrem expliziten Ziel? Und wenn ja, worauf bezieht sich dann der behauptete Funktionswandel? Tatsächlich zielt ja die CTA auf die Mitgestaltung oder Optimierung entstehender Technologien durch Stakeholder- und Nutzerlnnen-Beteiligung im Sinne des Gemeinwohls (Rip et al. 1995). Doch jenseits dieser recht abstrakten Gemeinsamkeit eines allgemeinen Gestaltungsanspruchs lassen sich deutliche Differenzen zwischen CTA und jener TA konstatieren, die sich im Rahmen einer anticipatory go- 
vernance herausbildet. Die CTA fokussiert in ihrem Modell aktiver Technikgestaltung auf Technologien, die so weit ausgereift sind, dass konkrete Anwendungen zum Gegenstand der TA gemacht werden können. In der Praxis bezieht sich CTA denn auch v. a. auf Industriebetriebe, in denen neue Technologien und deren praktische Anwendungen entwickelt werden. Im Kontext von emerging technologies wie der Nanotechnologie oder der synthetischen Biologie hingegen bleibt der Anwendungsbezug oft hypothetisch. TA ist auch hier aufgerufen, Technikentwicklung nicht mehr nur von außen zu untersuchen, sondern aktiv an deren Gestaltung teilzunehmen - jedoch nicht auf materialer (CTA), sondern gewissermaßen auf symbolischer Ebene: Es geht, wie erwähnt, um Deutungs- und Definitionsprozesse. Die TA richtet sich hier auf technowissenschaftliche Felder, die weder über fachliche oder disziplinäre Identität noch über einen präzisen Gegenstands- oder Anwendungsbereich verfügen.

Ein vergleichbarer Wandel scheint sich auch auf dem weiten Feld der STS abzuzeichnen. Dafür lassen sich eine Reihe von Indizien anführen. So haben in der letzten Zeit Reflexionen an Bedeutung gewonnen, die die Rolle sozialwissenschaftlicher Expertise für politische Entscheidungsprozesse thematisieren. Daraus hat sich mittlerweile eine Debatte entwickelt, die auf unterschiedlichen Ebenen, sowohl empirisch-deskriptiv als auch programmatisch, geführt wird. Ein vielbeachteter Beitrag ist in diesem Zusammenhang die Analyse und Programmatik von Collins und Evans (2002). Die beiden Autoren argumentieren in ihrem Artikel über die "Third Wave of Science Studies", dass STS gegenwärtig bereits zum zweiten Mal von einer grundlegenden Umwälzung betroffen sei. Der erste Wandel habe eine positivistische, objektivitätsgläubige Wissenschaftsforschung ab den 1970er Jahren in ihre konstruktivistische und dekonstruierende Phase geführt (maßgeblicher Bezugspunkt sind hier die sog. Laborstudien). Einen zweiten Wandel postulieren bzw. fordern die Autoren für die Gegenwart. Er solle die STS in eine Phase stärkerer politischer Relevanz versetzen. Voraussetzung dafür sei, dass wissenschaftlicher Expertise eine epistemische Sonderstellung zugestanden werde. In diesem Sinne geht es den Autoren um die Rekonstruktion einer klaren Grenzziehung zwischen Laien und Expertlnnen bzw. Politik (als Arena gesellschaftlicher Diskurse) und Technologie (als Arena des Faktischen). Dahinter steht der Anspruch, STS solle nicht nur über Geschichte reflektieren, sondern diese aktiv mitgestalten (,sociologists of scientific knowledge ... have a role to play in making history", ibid., S. 241).

Bei der Forderung allein ist es nicht geblieben. Es lässt sich vielmehr eine zunehmende Reflexion der praktischen Bedeutung von STS-Expertise für die 
Wissenspolitik (im Sinne von Stehr 2003) beobachten. Dies deutet darauf hin, dass in der STS-community das Bewusstsein zunimmt, über eine Akteurs-Position in diesem Feld zu verfügen. Ist es die Rolle von „ironists, reformers, or rebels" (Gisler/Schicktanz 2009), ist es die einer helfenden Hand oder einer Hilfswissenschaft (vgl. Burchell 2009)? Vor diesem Hintergrund lässt sich auch der gegenwärtig zu beobachtende Boom der Expertiseforschung verstehen. Im Vordergrund steht dabei der Versuch, auf Grundlage empirischer Studien Modelle der Verknüpfung von Wissen und Werten im Bereich der Forschungs- und Technologiepolitik zu entwickeln (z. B. Hoppe 2005). Zwecks Reflexion der politischen Bedeutung und Verwertung von Expertise werden Förderprogramme aufgelegt (z. B. das BMBF-Programm "Wissen für Entscheidungsprozesse"), es werden Zeitschriften gegründet (wie die seit 2008 erscheinende Zeitschrift für Politikberatung) und entsprechende Schwerpunktsetzungen in den Verlagsprogrammen vorgenommen, die sich in einem signifikanten Anstieg von Monographien, Überblicksdarstellungen und Handbüchern zur Politikberatung äußern (z. B. Weingart/ Lentsch 2008, Bröchler/Schützeichel 2008, Heidelberger Akademie der Wissenschaften 2006, Falk et al. 2006, Bogner/Torgersen 2005).

Ein weiteres Indiz für unsere These des Funktionswandels ergibt sich mit Blick auf die Institutionalisierung eines seltsam hybriden Feldes, nämlich der ELSI-Begleitforschung zu ethischen, rechtlichen und sozialen Aspekten von emergierenden Technologien in den 1990er Jahren. Das prominenteste Beispiel ist hier sicher das anlässlich der Initiative zur Erforschung des Humangenoms in den USA gestartete Programm. Dabei legte man implizit ähnlich wie in der frühen TA - ein lineares Modell von Technologieentwicklung zugrunde. Dementsprechend hatte die ELSI-Forschung von außen die gesellschaftlichen Folgen einer unabhängigen und ihren eigenen Gesetzen folgenden wissenschaftlich-technischen Entwicklung zu untersuchen. Dieses einfache Modell von Ursache-Wirkungsbeziehungen ist verschiedentlich kritisiert worden (Cook-Deegan 1994). Ausgehend davon entwickelten zuletzt Rabinow und Bennett (2008) eine Initiative, mittels anthropologischer Expertise Technowissenschaften und Begleitforschung zu integrieren und Grundlagen für eine erhöhte Reflexivität im Forschungsalltag bereitzustellen. In ähnlicher Weise zielt die "Laboratory Engagement Study" von Fisher (2007) auf Intervention in die Wissenschaftsgenese, und zwar vom Inneren der Technowissenschaft aus. In diesem Ansatz wird die Sozialwissenschaft mittels teilnehmender Beobachtung zu einem Organisator von entscheidungsrelevanter Reflexion im Forschungsalltag.

Aber auch die ELSI-Programme gehen heute neue Wege. Mit Blick auf die ELSI-Projekte im Rahmen des österreichischen Genomforschungspro- 
gramms GEN-AU lässt sich ein deutlicher Wandel konstatieren: Es geht nunmehr vorrangig um die zeitnahe Begleitung von Forschungs- und Technisierungsprojekten sowie die Gestaltung des öffentlichen Diskurses zu diesen Themen. Das vorrangige Ziel besteht darin, die Forschung zur Reflexion auf die ethischen oder sozialen Implikationen ihrer Praxis zu bewegen. In diesem Zusammenhang lässt sich feststellen, dass ELSI-Forschung auf die Expertise von STS zurückgreift und gleichzeitig die eher für TA typische Beratungsfunktion anstrebt. So ergeben sich zwischen STS, TA und ELSI deutliche Konvergenzen und vielfältige Überschneidungen hinsichtlich der Zielbestimmungen und Methoden sowie, nicht zuletzt, der beteiligten Institutionen.

\section{Resümee}

In diesem Text wurde argumentiert, dass Kontroversen um Wissenschaft und Technik je spezifische Herausforderungen für Governance darstellen. Ausgangspunkt war die Beobachtung, dass die Problematisierung von Technologien sich notwendigerweise innerhalb bestimmter Rahmungen vollzieht, wobei Risiko und Ethik die beiden prominentesten Fälle darstellen. Es sind diese kulturell geprägten Muster der Sinngebung, die einen bestimmten politischen Umgang mit Technologien implizieren, eben je nachdem, ob sie als Risiko- oder als Ethikproblem verstanden werden. Unter den Titeln von risk bzw. ethics governance wurde vorgeführt, welche Governance-Variationen sich in Abhängigkeit von der jeweiligen Rahmung ergeben. Dargestellt wurde dies konkret an den Beispielen von Expertise, Öffentlichkeitsbeteiligung und politischer Legitimation. Zu betonen bleibt, dass das Verhältnis von Rahmung und Governance als ein wechselseitig sich bedingendes zu denken ist - und nicht als einseitiges Determinationsverhältnis, in welcher Form auch immer. So wie eine bestimmte Rahmung ganz bestimmte politische Verfahrensformen und Begründungsmuster als legitim erscheinen lässt, sind auch umgekehrt die jeweils aktualisierten Governance-Formen für die Stabilisierung bzw. Modifikation des Rahmens relevant.

Wir sind davon ausgegangen, dass neu entstehende Technologien, die aktuell in dem noch recht anspruchslosen Begriff der emerging technologies gefasst werden, in erster Linie als diskursives Phänomen verstanden werden müssen. Auf politischer Ebene gilt die Sorge einer vorsorglichen Auseinandersetzung mit denkbaren Konfliktpotenzialen. Vor diesem Hintergrund artikulieren sich neuartige Ansprüche und Erwartungen, die sich im Prinzip 
sowohl auf Beteiligungsformate und Verfahrensformen, aber auch auf die in diesen Prozessen relevante Expertise beziehen. Dies wurde als Übergang zu einer antizipatorischen Governance diagnostiziert.

In theoretischer Hinsicht zielt unser Beitrag also darauf ab, dem in der Literatur noch recht vagen Begriff einer anticipatory governance schärfere Konturen zu verleihen - wobei auf der Hand liegt, dass dies erst den Einstieg in eine notwendige Debatte bedeutet. Immerhin wurde im Rekurs auf Expertise ein Governance-relevanter Bereich vorgeführt, in dem aufgrund veränderter Zeitstrukturen sich wandelnde Rollenerwartungen bemerkbar werden. In praktischer Hinsicht reagieren unsere Befunde kritisch auf die im politischen Diskurs prominente Unterstellung, man könne die Erfahrungen mit vergangenen Kontroversen umstandslos auf die Zukunft übertragen. Grundlegende Voraussetzung für ein "Lernen aus vergangenen Konflikten" ist die Differenzierung zwischen Technikkontroversen, die in diesem Text entlang der Unterscheidungen von real/antizipiert und Risiko/Ethik vorgenommen wurde. Anticipatory governance muss gerade deshalb als problematisch gelten, weil nicht vorhergesagt werden kann, innerhalb welcher Rahmen Kontroversen über zukünftige Technologien ausgetragen werden. Jede Intervention wird selbst zu einer Beteiligung an dieser kontingenten Entwicklung.

Der diagnostizierte Funktionswandel sozialwissenschaftlicher Expertise gründet sich letztlich auf einem Wandel von Konflikt- und Politikmustern im Zuge veränderter Formen und Vorstellungen von Wissenschafts- und Technikentwicklung. Sozialwissenschaftliche Expertise - sei es in den Formaten von TA, ELSI oder STS - sieht sich gegenwärtig mit neuen Rollenerwartungen konfrontiert: Sie wird auf diese Weise zu einer bewussten (und damit auch verantwortungspflichtigen) Mitspielerin in der politischen Gestaltung von realen oder antizipierten Kontroversen um Wissenschaft und Technik.

\section{Anmerkungen}

1 Rick Weiss: „For Science, Nanotech Poses Big Unknowns", Washington Post, 1 February 2004, S. A01.

2 Anna Fazackerley: „Nanotechnology won't suffer GM fate“, Times Higher Education Supplement, 27 June 2003, http://www.timeshighereducation.co.uk/story.asp?sec tioncode $=26 \&$ storycode $=177714$ (abgerufen am 14. 7. 2009).

3 "'Risk' is the only game in town", wie ein Gutachter dieses Artikels treffend anmerkt. Die Dominanz des Risikodiskurses steht dabei in Wechselwirkung mit dem vorhandenen Regulierungsinventar (Regulierungsbehörden und -instrumente), das ebenfalls auf Risikoevidenzen fokussiert. 
4 "Ich bin kein Briefträger, der Botschaften überbringt" - Gerhard Schröder im Interview; http://www.tagesspiegel.de/politik/art771,1967538 (abgerufen am 8.8. 2006).

5 Der Bezug zwischen Rahmen und Governance-Strukturen kann als wechselseitig gedacht werden. Bestimmte Rahmen bestärken die mit ihnen kompatiblen Governance-Strukturen, indem sie ihnen Legitimität zusprechen. Die Entwicklung bestimmter Governance-Strukturen wiederum befördert die mit ihnen kompatiblen Rahmen, weil die in diesen Rahmen operierenden Argumente unmittelbar in konkrete steuerungspolitische Forderungen übersetzbar sind.

6 Man vergleiche dazu etwa das Deutsche Mobilfunk Forschungsprogramm (DMF) des deutschen Bundesamtes für Strahlenschutz. Es verfolgt vier parallele Forschungsstränge: Biologie, Epidemiologie, Dosimetrie und Risikokommunikation. Nur in letzterem Bereich tauchen die Sozialwissenschaften auf (http://www.emf-forschungsprogramm. de/forschung).

7 Eine Aufweichung dieser Prinzipien, etwa im Kontext des Vorsorgeprinzips (vgl. von Schomberg 2005), kann daher als Indiz für einen Frame-Wandel gesehen werden.

8 "Ich habe nichts gegen grüne Gentechnik", in: Frankfurter Allgemeine Zeitung, 18. 4. 2009; http://www.faz.net/s/Rub594835B672714A1DB1A121534F010EE1/ Doc E2E172490B21D4065A0B82A067E105840 ATpl Ecommon Scontent.html (abgerufen am 28. 8. 2009).

9 Jörg Albrecht/Volker Stollorz: „Kleine Käfer, große Fragen. Ist Genmais gefährlich?“ Frankfurter Allgemeine Sonntagszeitung, 19. 4. 2009, http://www.faz.net/s/Rub 80665A3C1FA14FB9967DBF46652868E9/Doc E5C0249F49858443FADC2C9C8 A73A0999 ATpl Ecommon Scontent.html (abgerufen am 20. 4. 2009).

10 „Brown compromise over embryo vote“, in: BBC News, http://news.bbc.co.uk/2/hi/ uk_news/politics/7312715.stm (abgerufen am 20. 10. 2009).

11 Die synthetische Biologie versucht, analytische Erkenntnisse der Biologie (etwa über Genome) in ingenieursmäßiger Weise in Konstruktionen umzusetzen. Es geht um praktische Anwendungen, aber auch um die Herstellung von Organismen zum Erkenntnisgewinn.

12 Empirische Analysen zu Beteiligungsexperimenten im Bereich der Nanotechnologie zeigen allerdings, dass sich die damit erhofften Demokratisierungs- oder Rationalisierungspotenziale bisher nicht realisiert haben (Kurath/Gisler 2009).

13 Tony Blair warnt etwa in einer Rede vor der Royal Academy of Sciences 2002, dass die Leistungen der Wissenschaft nur erfolgreich umgesetzt werden könnten, wenn der (implizite) Vertrag zwischen Wissenschaft und Öffentlichkeit neu formuliert würde. (http:/ /www.dius.gov.uk/science/science_and_society/public_engagement/public_attitudes 2005, abgerufen am 11.10. 2009).

14 Vgl. etwa das 2002 ins Leben gerufene "Expectations Network", siehe im Netz unterhttp://www.york.ac.uk/res/satsu/expectations/about.htm. (abgerufen am 5.10. 2009)

15 Auch in diesem Fall muss es aussichtslos bleiben, so etwas wie einen Ursprung des Begriffs bestimmen zu wollen. Immerhin lässt sich eine allmähliche Durchsetzung dieses Begriffs ab der Jahrtausendwende beobachten. Karinen und Guston (2009) unterscheiden drei Verwendungskontexte, nämlich die Umweltwissenschaften, Studien zu öffentlicher Verwaltung und Management sowie ELSA-Forschung zu Nanotechnologie. Vgl. zu einer ersten Verwendung in diesem Kontext: Guston/Sarewitz 
Alexander Bogner, Karen Kastenhofer, Helge Torgersen

(2002).

16 Dieser Widerspruch wurde bereits in Zusammenhang mit der Ausformulierung des Vorsorgenprinzips eingehender diskutiert (van Asselt/Vos 2006).

17 "Nanotechnology - the ability to understand and control matter with ultimate precision - is the most powerful and enabling technology humankind has ever developed. Nanotechnology is used to create materials, devices and systems with fundamentally new properties and functions that will change the world as we know it." Ankündigungstext für eine hochkarätig besetzte Tagung an der Cornell University am 14. Juni 2007 (http://www.cnf.cornell.edu/cnf_nanofutures.html, zuletzt abgerufen am 28. 9. 2009).

18 So etwa die kritische, aber häufig zitierte Action Group on Erosion, Technology and Concentration (ETC Group) unterhttp://www.etcgroup.org/en/issues/bangconvergence.html. (abgerufen am 1. 10. 2009)

19 Bernhard Epping: „Synthetische Biologie. Frankensteins Zeit ist gekommen“, Der Spiegel, 27. 12. 2008, http://www.spiegel.de/wissenschaft/mensch/0,1518,596579, 00.html (abgerufen am 5. 8. 2009).

20 Das OTA wurde 1995 im Sinne der damals dominanten konservativen Strömung als entbehrlich angesehen und geschlossen.

Literatur

Abels, Gabriele. 2009. Organizer, observer and participant - What role for social scientists in different pTA models? Science, Technology \& Innovation Studies 5: 19-35.

Abels, Gabriele, und Alfons Bora. 2004. Demokratische Technikbewertung. Bielefeld: transcript.

Bauer, Martin W., und George Gaskell, Hg. 2002. Biotechnology: The Making of a Global Controversy. Cambridge: Cambridge University Press.

Barben, Daniel, Erik Fisher, Cynthia Selin, und David H. Guston. 2008. Anticipatory Governance of Nanotechnology: Foresight, Engagement, and Integration. In: The Handbook of Science and Technology Studies, hrsg. Edward J. Hackett, Olga Amsterdamska, Michael Lynch und Judy Wajcman, 979-1000. Cambridge, MA: MIT Press.

Bogner, Alexander. 2009. Ethisierung und die Marginalisierung der Ethik. Soziale Welt 60: 119-137.

Bogner, Alexander. 2010. Partizipation als Laborexperiment. Paradoxien der Laiendeliberation in Technikfragen. Zeitschrift für Soziologie 39: 87-105.

Bogner, Alexander, und Helge Torgersen, Hg. 2005. Wozu Experten? Ambivalenzen der Beziehung von Wissenschaft und Politik. Wiesbaden: VS.

Bogner, Alexander, Karen Kastenhofer, und Helge Torgersen, Hg. 2009. Inter- und Transdisziplinarität im Wandel? Neue Perspektiven auf problemorientierte Forschung und Politikberatung. Baden-Baden: Nomos.

Bröchler, Stefan, und Rainer Schützeichel, Hg. 2008. Politikberatung. Stuttgart: Lucius \& Lucius (UTB). 
Burchell, Kevin. 2009. A helping hand or a servant discipline? Interpreting non-academic perspectives on the roles of social science in participatory policy-making. Science, Technology \& Innovation Studies 5: 49-61.

Bush, Vannevar. 1945. Science: The Endless Frontier. A Report to the President. Washington: United States Government Printing Office.

Collingridge, David. 1981. The Social Control of Technology. Milton Keynes: Open University Press.

Collins, Harry M., und Robert Evans. 2002. The Third Wave of Science Studies: Studies of Expertise and Experience. Social Studies of Science 32: 235-296.

Cook-Degan, Robert. 1994. The Gene Wars: Science, Politics, and the Human Genome. New York: Norton.

De Marchi, Bruna. 2003. Public participation and risk governance. Science and Public Policy 30(3): 171-176.

Europäische Kommission. 2001. Europäisches Regieren. Ein Weißbuch. Brüssel: Europäische Kommission.

Falk, Svenja, Andrea Römmele, Dieter Rehfeld, und Martin Thunert, Hg. 2006. Handbuch Politikberatung. Wiesbaden: VS.

Feenberg, Andrew. 1999. Questioning Technology. London/New York: Routledge.

Fiedeler, Ulrich. 2008. Technology Assessment of Nanotechnology: Problems and Methods in Assessing Emerging Technologies. In: Excavating Futures of Nanotechnology. The Yearbook of Nanotechnology in Society, hrsg. Erik Fisher, Cynthia Selin und Jameson Wetmore, 241-263. New York: Springer.

Fisher, Erik. 2007. Ethnographic Intervention: Probing the Capacity of Laboratory Decisions. NanoEthics 1: 155-165.

Gaskell, George, Agnes Allansdottir, Nick Allum, et al. 2006. Europeans and Biotechnology in 2005: Patterns and Trends, Final report on Eurobarometer 64.3. Brüssel: European Commission Directorate-General for Research.

Gehlen, Arnold. 2007. Die Seele im technischen Zeitalter. Sozialpsychologische Probleme in der industriellen Gesellschaft. Frankfurt/M.: Klostermann.

Giersch, Herbert, Hg. 1982. Emerging technologies: consequences for economic growth, structural change, and employment: symposium 1981. Tübingen: Mohr Siebeck.

Gill, Bernhard. 2003. Streitfall Natur. Weltbilder in Technik- und Umweltkonflikten. Wiesbaden: Westdeutscher Verlag.

Gisler, Priska, und Silke Schicktanz. 2009. Ironists, reformers, or rebels? Reflections on the role of the social sciences in the process of science policy making. Science, Technology \& Innovation Studies 5: 5-17.

Goffman, Erving. 1993. Rahmen-Analyse. Ein Versuch über die Organisation von Alltagserfahrungen. 3. Aufl., Frankfurt/M.: Suhrkamp.

Grin, John, und Armin Grunwald, Hg. 2000. Vision Assessment: Shaping Technology in 21 st Century Society. Towards a Repertoire for Technology Assessment, Wissenschafts- 
Alexander Bogner, Karen Kastenhofer, Helge Torgersen

ethik und Technikfolgenbeurteilung. Berlin: Springer.

Guston, David H., und Daniel Sarewitz. 2002. Real-time technology assessment. Technology in Society 24: 93-109.

Heidelberger Akademie der Wissenschaften, Hg. 2006. Politikberatung in Deutschland. Wiesbaden: VS.

Hoppe, Robert. 2005. Rethinking the science-policy nexus: from knowledge utilization and science technology studies to types of boundary arrangements. Poiesis \& Praxis 3: 199-215.

House of Lords. 2000. Science and Society. Select Committee on Science and Technology Third Report (HL 38). London: House of Lords.

IRGC (International Risk Governance Council). 2006. Nanotechnology Risk Governance. Recommendations for a global, coordinated approach to the governance of potential risks. Geneva: IRGC.

Irwin, Allan. 2001. Constructing the scientific citizen: science and democracy in the biosciences. Public Understanding of Science 10: 1-18.

Joss, Simon. 1995. Consensus conferences and their contribution to science policy. Science, Technology and Innovation 8: 14-19.

Karinen, Risto, und David H. Guston. 2009. Toward Anticipatory Governance: The Experience with Nanotechnology. In: Governing Future Technologies: Nanotechnology and the Rise of an Assessment Regime, hrsg. Mathias Kaiser, Monika Kurath und Christoph Rehmann-Sutter, 217-232. Dordrecht, NL: Springer.

Kastenhofer, Karen. 2009a. Debating the risks and ethics of emerging technosciences. Innovation: The European Journal of Social Science Research 22: 77-103.

Kastenhofer, Karen. 2009b. Zwischen "schwacher" und "starker" Interdisziplinarität: Sicherheitsforschung zu neuen Technologien. In: Inter- und Transdisziplinarität im Wandel? Neue Perspektiven auf problemorientierte Forschung und Politikberatung, hrsg. Alexander Bogner, Karen Kastenhofer und Helge Torgersen, 87-122. Baden-Baden: nomos.

Kurath, Monika, und Priska Gisler. 2009. Informing, involving or engaging? Science communication in the ages of atom-, bio- and nanotechnology. Public Understanding of Science 18: 559-573.

Lau, Christoph. 1991. Neue Risiken und gesellschaftliche Konflikte. In: Politik in der Risikogesellschaft, hrsg. Ulrich Beck, 248-266. Frankfurt/M.: Suhrkamp.

Lindsey, Nicola, Mercy W. Kamara, Erling Jelsoe, und Arne Thing Mortensen. 2001. Changing Frames: the Emerging of Ethics in European Policy on Biotechnology. Politeia 17: 80-93.

Maasen, Sabine. 2002. Die gesellschaftliche Disziplinierung bio- und gen-ethischer Fragen durch die politische Institutionalisierung von "Diskurs". Expertise im Auftrag des BMBF <www.sciencepolicystudies.de/dok/expertise-maasen.pdf> (abgerufen am 20. 10. 2009).

Mayntz, Renate. 2009. Über Governance. Institutionen und Prozesse politischer Rege- 
Antizipierte Technikkontroversen als Governance-Problem

lung. Frankfurt/New York: Campus.

Michael, Mike. 1996. Ignoring science: discourses of ignorance in the public understanding of science. In: Misunderstanding science? The public reconstruction of science and technology, hrsg. Alan Irwin und Brian Wynne, 107-125. Cambridge: Cambridge University Press.

Nelkin, Dorothy, Hg. 1984. Controversy, Politics of Technical Decisions. 2. Aufl. Newbury Park/CA: Sage.

Nennen, Heinz-Ulrich, und Detlef Garbe, Hg. 1996. Das Expertendilemma - Zur Rolle wissenschaftlicher Gutachter in der öffentlichen Meinungsbildung. Berlin: Springer.

Nisbet, Matthew C., und Bruce V. Lewenstein. 2002. Biotechnology and the American Media. The Policy Process and the Elite Press, 1970 to 1999. Science Communication 23: 359-391.

Nordmann, Alfred. 2004. Was ist TechnoWissenschaft? - Zum Wandel der Wissenschaftskultur am Beispiel von Nanoforschung und Bionik. In: Bionik: Aktuelle Forschungsergebnisse in Natur-, Ingenieur- und Geisteswissenschaft, hrsg. Torsten Rossmann und Cameron Tropea, 209-218. Berlin: Springer.

Porter, Alan L. 1995. Technology Assessment. Impact Assessment 13: 135-151.

Rabinow, Paul, und Gaymon Bennett. 2008. Ars Synthetica, Designs for Human Practices. Houston: Rice University Press.

Rip, Arie. 2006. Folk Theories of Nanotechnologists. Science as Culture 15: 349-365.

Rip, Arie, Thomas J. Misa, und Johan Schot, Hg. 1995. Managing Technology in Society. The Approach of Constructive Technology Assessment. London/New York: Pinter.

Roco, Mihail C., und William Sims Bainbridge, Hg. 2002. Converging Technologies for Improving Human Performance. Nanotechnology, Biotechnology, Information Technology and Cognitive Science. Arlington, Virginia: NSF/DOC.

Saretzki, Thomas. 1999. TA als diskursiver Prozeß. In: Handbuch Technikfolgenabschätzung, hrsg. Stephan Bröchler, Georg Simonis und Karsten Sundermann, 641-653. Berlin: Sigma.

Schön, Donald A., und Martin Rein. 1994. Frame Reflection: Toward the Resolution of Intractable Policy Controversies. New York: Basic Books.

Schummer, Joachim. 2006. Cultural diversity in nanotechnology ethics. Interdisciplinary Science Reviews 31: 217-230.

Sclove, Richard E. 1995. Democracy and Technology. New York/London: The Guildford Press.

Selin, Cynthia. 2007. Expectations and the Emergence of Nanotechnology. Science, Technology \& Human Values 32: 196-220.

Simmel, Georg. 1958. Der Streit. In: Soziologie. Untersuchungen über die Formen der Vergesellschaftung, ders., 186-255. Berlin: Duncker \& Humblot.

Snow, Charles Percy. 1963. Die zwei Kulturen. Literarische und naturwissenschaftliche Intelligenz. Stuttgart: Klett. 
Alexander Bogner, Karen Kastenhofer, Helge Torgersen

Stehr, Nico. 2003. Wissenspolitik: Die Überwachung des Wissens. Frankfurt/M.: Suhrkamp.

van Asselt, Marjolein B. A., und Ellen Vos. 2006. The Precautionary Principle and the Uncertainty Paradox. Journal of Risk Research 9: 313-336.

von Blumenthal, Julia. 2005. Governance - eine kritische Zwischenbilanz. Zeitschrift für Politikwissenschaft 15: 1149-1180.

van den Daele, Wolfgang. 1993. Sozialverträglichkeit und Umweltverträglichkeit, Inhaltliche Mindeststandards und Verfahren bei der Beurteilung neuer Technik. Politische Vierteljahresschrift 34: 219-248.

van den Daele, Wolfgang, Alfred Pühler, und Herbert Sukopp. 1996. Grüne Gentechnik im Widerstreit. Modell einer partizipativen Technikfolgenabschätzung zum Einsatz transgener herbizidresistenter Pflanzen. Weinheim: VCH Verlagsgesellschaft.

van Eijndhoven, Josée C. M. 1997. Technology Assessment: Product of Process? Technology Forecasting and Social Change 54: 269-286.

von Schomberg, René. 2005. Die normativen Dimensionen des Vorsorgeprinzips. In: Risikoregulierung bei unsicherem Wissen: Diskurse und Lösungsansätze, hrsg. Leonhard Hennen und Arnold Sauter, 91-118. Berlin: Büro für Technikfolgen-Abschätzung.

Weingart, Peter, und Justus Lentsch. 2008. Wissen - Beraten - Entscheiden: Form und Funktion wissenschaftlicher Politikberatung in Deutschland. Weilerswist: Velbrück Wissenschaft.

Zürn, Michael. 2007. Was heißt, Governance'? Gegenworte, Hefte für den Disput über Wissen 18: 28-31. 